\title{
Prevalencia de alteraciones en la interfase vitreoretiniana detectadas mediante tomografía de coherencia óptica de dominio espectral
}

\author{
Julie Jacob Peter Stalmans \\ Universidad Ziekenhuis Leuven, Departamento de Oftalmología, University Hospitals Leuven, Lovaina, Bélgica
}

\section{Palabras clave}

Cambio en la interfase vitreomacular - Tomografía de coherencia óptica. Adhesión vitreomacular . Desprendimiento de vítreo posterior · Tracción vitreomacular

\section{Resumen}

Objetivo: El objetivo principal del presente estudio consistió en determinar la prevalencia de los cambios en la interfase vitreomacular (IVM) mediante tomografía de coherencia óptica (OCT) en la población general. En segundo lugar, se describieron otros cambios de la OCT. Métodos: Las anomalías en la IVM se diagnosticaron mediante OCT y se distribuyeron de acuerdo con la clasificación del grupo International Vitreomacular Traction Study (estudio internacional de tracción vitreomacular, IVTS, por sus siglas en inglés) y se dividieron en 3 grados según John et al. [Retina 2014;34:442-446]. Resultados: La prevalencia calculada de anomalías vitreomaculares en la población belga de $\geq 50$ años fue del 1,17\% [intervalo de confianza (IC 0,38-3,62)] en el caso de tracción vitreomacular (TVM) focal de grado 1 ; del $0,39 \%$ (IC 0,052,76 ) en el caso de TVM focal de grado 2; del 8,17\% (IC 5,33$12,53)$ en el caso de adhesión vitreomacular focal; y del $17,9 \%$ (IC 13,41-23,9) en el caso de adhesión vitreomacular difusa. Conclusiones: Se presentó la prevalencia de anomalías vitreomaculares en un estudio de cohortes belga. Estos

\section{KARGER}

๑ 2017 S. Karger AG, Basel

E-Mail karger@karger.com

www.karger.com/oph resultados concuerdan en gran medida con los datos presentados previamente sobre la prevalencia de la TVM. Un conocimiento correcto sobre la epidemiología de las alteraciones en la IVM y un diagnóstico temprano permitirán una intervención satisfactoria.

(c) 2017 S. Karger AG, Basel

\section{Introducción}

Los cambios en la interfase vitreomacular (IVM) relacionados con la edad comprenden adhesión vitreomacular (AVM), donde el vítreo se separa de la mácula aunque sigue adherido a la fóvea (primer estadio de la formación del desprendimiento de vítreo posterior [DVP]), tracción vitreomacular (TVM), en el que el vítreo se licua y se separa de la mácula y una adhesión anormalmente estrecha entre el vítreo y la mácula crea una distorsión anatómica de la superficie de la retina, formación de agujeros maculares (AM) cuando un aumento de la tracción vítrea separa todas las capas de la retina en la fóvea $[1,2], y$ membranas epirretinianas, que consisten en proliferaciones en la unión vitreoretiniana tras un DVP [3]. A

The Spanish version should be cited as DOI: 10.1159/000477511. The original version is in English and should be cited as DOI: $10.1159 / 000446585$.
Dr. Julie Jacob

Departamento de Oftalmología, University Hospitals Leuven Herestraat 49

BE-3000 Lovaina (Bélgica)

E-Mail julie.jacob@uzleuven.be 
medida que el ojo envejece, el vítreo casi siempre se separa de la retina por un desprendimiento de vítreo posterior $[4,5]$, pero si esta separación no es completa puede conllevar una pérdida o distorsión de la visión. La AVM y la TVM conforman dos de las muchas situaciones que pueden ocurrir durante el transcurso de un desprendimiento de vítreo posterior incompleto [6]. Pueden contribuir al desarrollo o empeoramiento de enfermedades graves, tales como degeneración macular húmeda relacionada con la edad y edema macular diabético [7-9]. Se ha publicado que la resolución espontánea de la TVM varía entre el 11 y el 53\%; sin embargo, estas series contenían un número limitado de pacientes [10-13]. Se ha publicado que las membranas epirretinianas permanecen estables en el $38,8 \%$ de los pacientes y que remiten en el $25,7 \%$ de los pacientes [4].

No obstante, si las anomalías en la IVM no son diagnosticadas o tratadas pueden provocar graves enfermedades con mal pronóstico.

Aunque la prevalencia [14-18] y la incidencia [19] de los AM en la población general se encuentran bien documentados se disponen de pocos datos sobre la prevalencia de la AVM y la TVM en la población general $[20,21]$. El diagnóstico de las enfermedades en la IVM a menudo se realiza tan solo en estadios avanzados, cuando los pacientes experimentan la carga de la enfermedad sobre la vista en las últimas etapas de la enfermedad. Hoy en día los instrumentos de diagnóstico avanzados permiten obtener una mejor comprensión de la prevalencia de las alteraciones en la IVM en estadios tempranos [1]. Además, junto con las nuevas opciones de tratamiento, resulta posible que las estrategias terapéuticas más tempranas y mejores para alteraciones en la IVM reduzcan la incidencia de progresión hacia complicaciones más graves [22].

La tomografía de coherencia óptica (OCT, por sus siglas en inglés) permite visualizar la IVM y ha contribuido a que se pueda comprender mejor. La OCT tiene el potencial de permitir un diagnóstico precoz con un tratamiento subsiguiente de las alteraciones en la IVM [1].

Dado que la AVM tiene un papel esencial en la progresión de las alteraciones en la IVM, la liberación de la adhesión puede mejorar el pronóstico. Hasta hace muy poco, el tratamiento de referencia de las adhesiones implicaba una vitrectomía pars plana con el objetivo de eliminar el vítreo del ojo mediante cirugía. Esta técnica resultaba satisfactoria, pero la cirugía es invasiva e incómoda para el paciente y está asociada a complicaciones, tales como sangrado, infección, edema macular cistoide, formación de cataratas y desprendimiento de retina [23].
En 2012, la FDA aprobó el uso de ocriplasmina (Jetrea ${ }^{\circledR}$, Thrombogenics NV) - un agente biológico para el tratamiento no invasivo de adhesiones. La ocriplasmina es una inyección intravítrea que se administra para el tratamiento de la TVM con o sin AM de espesor total concomitante $[22,24]$. La ocriplasmina rompe la fibronectina y la laminina, dos componentes de la IVM que actúan como pegamento para mantener la interfase unida. La ocriplasmina provoca una vitreolisis farmacológica, en la que el vítreo se licua y se desprende de la retina [22]. La ocriplasmina es un tratamiento generalmente inocuo y que se tolera bien [25], e implica un menor riesgo de desprendimiento de retina y cataratas que la vitrectomía; no obstante, conlleva nuevas complicaciones, tales como reducción aguda transitoria de la agudeza visual, acumulación de fluido subretiniano, cambios externos en la retina y cambios en el grosor macular [25-28].

La disponibilidad del tratamiento farmacológico que permite una intervención temprana requiere un conocimiento suficiente de la epidemiología de las alteraciones en la IVM.

\section{Objetivos y métodos}

El objetivo principal del presente estudio fue determinar la prevalencia de los cambios en la IVM mediante OCT en la población general.

\section{Diseño del estudio}

El estudio aprovechó la oportunidad del «Day of Science». Se trata de una iniciativa promovida por el gobierno flamenco para invitar al público general a universidades y hospitales en Flandes y Bruselas (Bélgica) y tiene como propósito hacer accesibles los temas científicos. Este acontecimiento se ha celebrado anualmente durante varios años y atrae a decenas de miles de visitantes. Technopolis (www.technopolis.be) coordina la iniciativa «Day of Science» en colaboración con universidades, instituciones y asociaciones científicas flamencas, museos, empresas y federaciones.

El estudio tuvo lugar en el Departamento de Oftalmología de la Universidad Ziekenhuis Leuven (UZ Leven), University Hospitals Leuven, Lovaina, Bélgica, durante la cuarta edición anual del «Day of Science» que se celebró el 23 de noviembre de 2013. El Departamento de Oftalmología de la Universidad Hospitals Leuven organizó una sesión informativa sobre el desarrollo de fármacos para tratar enfermedades de la IVM en colaboración con Thrombogenics, en la que se les dio a los visitantes la oportunidad de someterse a una OCT de ojos. Después, pudieron discutir el resultado con un oftalmólogo y asistir a una sesión informativa sobre enfermedades de la IVM.

\section{Indicaciones clínicas}

No se especificó ninguna indicación clínica para el estudio, pues el objetivo comprendía evaluar la prevalencia entre la población general. El criterio de exclusión del estudio se basó en la presencia de medios opacos. 


\section{Cohorte del estudio}

La cohorte comprendió 481 personas, de las cuales el 55\% eran mujeres (media de edad 48 años) y el $45 \%$ hombres (media de edad 52 años; $\mathrm{p}=0,1687$ ). Los criterios de inclusión eran visitantes hombres y mujeres de $>18$ años de edad con medios transparentes. Se obtuvo el consentimiento informado por escrito de todos los visitantes antes de que fueran incluidos en el estudio en cumplimiento del reglamento pertinente a los ensayos clínicos. Todos los visitantes tuvieron la oportunidad de discutir el resultado de su OCT con un oftalmólogo. En caso de recibir un resultado anómalo, se le recomendaba al visitante acudir al oftalmólogo del Departamento de Oftalmología. Todos los visitantes recibieron una copia impresa de su OCT. La cohorte se dividió en dos grupos de $<50$ y $\geq 50$ años de edad antes de llevar a cabo el análisis de acuerdo con la normalización de edad de la población mundial de la OMS [29].

\section{Técnicas}

Las OCT se realizaron con cinco máquinas de OCT Cirrus 5000 (Zeiss), aplicando un protocolo normalizado de OCT de 5 exploraciones de alta definición del área foveal en ambos ojos de cada visitante. El programa utilizado para interpretar las imágenes era el complemento Zeiss Viewer (el programa que viene por defecto en todas las máquinas Zeiss). Se creó un archivo electrónico para cada visitante, y las imágenes OCT se almacenaron en el servidor DICOM con firewall del hospital. Este procedimiento es idéntico al que se usa con pacientes habituales del Departamento de Oftalmología para garantizar la seguridad de sus datos. Se registraron los datos personales, que comprendían el sexo y la edad, así como los antecedentes médicos y oftalmológicos de interés. Los datos se analizaron de manera anónima.

La clasificación y la distribución por grados de las OCT se llevó a cabo de acuerdo con el sistema de clasificación del gupo Inter-

national Vitreomacular Traction Study (estudio internacional de tracción vitreomacular, IVTS, por sus siglas en inglés) [1]:

- Se consideró que existían cambios en la IVM cuando la corteza del vítreo posterior se hubiera desprendido de la superficie de la retina pero permaneciera adherida al menos en un lugar dentro de un radio de $3 \mathrm{~mm}$ de la fóvea.

- Si existía alguna adhesión pero no había ningún cambio discernible en la anatomía de la retina se consideraba que el ojo tenía únicamente AVM.

- Si se observaba alguna tracción vitreomacular, derecho hacia el vítreo o levantado tangencialmente y alejado de su ubicación original, se consideraba TVM.

- La TVM se clasificaba además como difusa $(<1.500 \mu \mathrm{m}$ de área de adhesión y distorsión asociada) o focal ( $\leq 1.500 \mu \mathrm{m}$ de área de adhesión y distorsión asociada), definido según el Grupo ITVTS.

- La TVM se clasificaba además de grado 1 (distorsión de la superficie foveal, de grado 2 (con formación pseudoquística), y de grado 3 (presencia de fluido subretiniano), según la clasificación propuesta por John et al. [10].

La clasificación de las membranas epirretinianas en OCT se realizó de acuerdo con el estudio Blue Mountains Eye [15], y el pliegue macular se definió como la señal hiperreflectiva en la superficie interna de la retina con evidencia de distorsión de la retina interna. La maculopatía en celofán se definió como un aumento en la reflectividad de la superficie interna de la retina sin distorsión.

La refracción se midió utilizando un focómetro.

Prevalencia de alteraciones en la interfase vitreoretiniana
Marco de análisis estadístico

Se llevó a cabo un análisis estadístico con el programa comercial SAS $^{\circledR}$ (Sistema de Análisis Estadístico, The SAS Institute, Cary, N.C., EE. UU.) para Windows, version 9.3.

Las variables continuas se presentaron como la media con desviación estándar, mínimo, máximo e intervalo de confianza (IC) del 95\% con la hipótesis de normalidad. Las variables binarias se presentaron como números y porcentajes, con un IC de Clopper-Pearson (exacto) del 95\%. Las variables polinomiales se presentaron como números con porcentajes por categoría.

Para comparar estadísticamente variables entre grupos, se utilizó la prueba del orden de la suma de Wilcoxon para las variables continuas, la prueba exacta de Fisher para las variables binarias, y la prueba Cochran-Mantel-Haenszel para las variables polinomiales. Se estudió la influencia de la edad, el sexo, el estado refractivo y el hecho de haberse sometido a cirugía previa sobre las tasas de prevalencia de los cambios en la IVM.

Para obtener una estimación más precisa de los datos de prevalencia, se calcularon IC de Poisson del 95\% adicionales.

\section{Cumplimiento}

El estudio se llevó a cabo de conformidad con los principios de la Directiva de la Unión Europea sobre Ensayos Clínicos (2001/20/ ED) y todos los requisitos locales/regionales necesarios para cumplir con las disposiciones de la Declaración de Helsinki (Asociación Médica Mundial, Edinburgo, 2000). El Comité de Ética del University Hospitals Leuven aprobó el estudio antes de que comenzara.

\section{Resultados}

\section{Características demográficas y otras características iniciales}

La cohorte comprendió 481 personas. Se excluyó a dos personas del análisis detallado: 1 persona no se sometió a la OCT después de haber firmado el consentimiento informado, y en el caso de 1 persona, la OCT se realizó de forma errónea en la arcada superior. 479 visitantes adultos y 956 ojos (2 visitantes eran monoftálmicos) permanecieron en el grupo del estudio (fig. 1).

En total, 479 sujetos (956 ojos) fueron incluidos en el estudio, de los cuales el 55\% eran mujeres y el $45 \%$ hombres $(\mathrm{p}=0,1687)$. El intervalo de edad era de 34 a 66 años. El $53.65 \%$ de la población del estudio era de $\geq 50$ años. El equivalente esférico medio del grupo de estudio era $-0,42 \pm 2,65$. Un total de 20 sujetos (36 ojos) eran pseudofáquicos.

\section{Resultados clínicos}

Del total de 956 ojos, 146 poseían anomalías en la IVM. Para el análisis de población, solo 1 ojo por paciente se incluyó tras la aleatorización.

En toda la cohorte del estudio, hubo 3 ojos con TVM focal en grado $1(0,63 \%), 1$ ojo con TVM focal en grado

Ophthalmologica 2017;238(suppl 1):9-15 


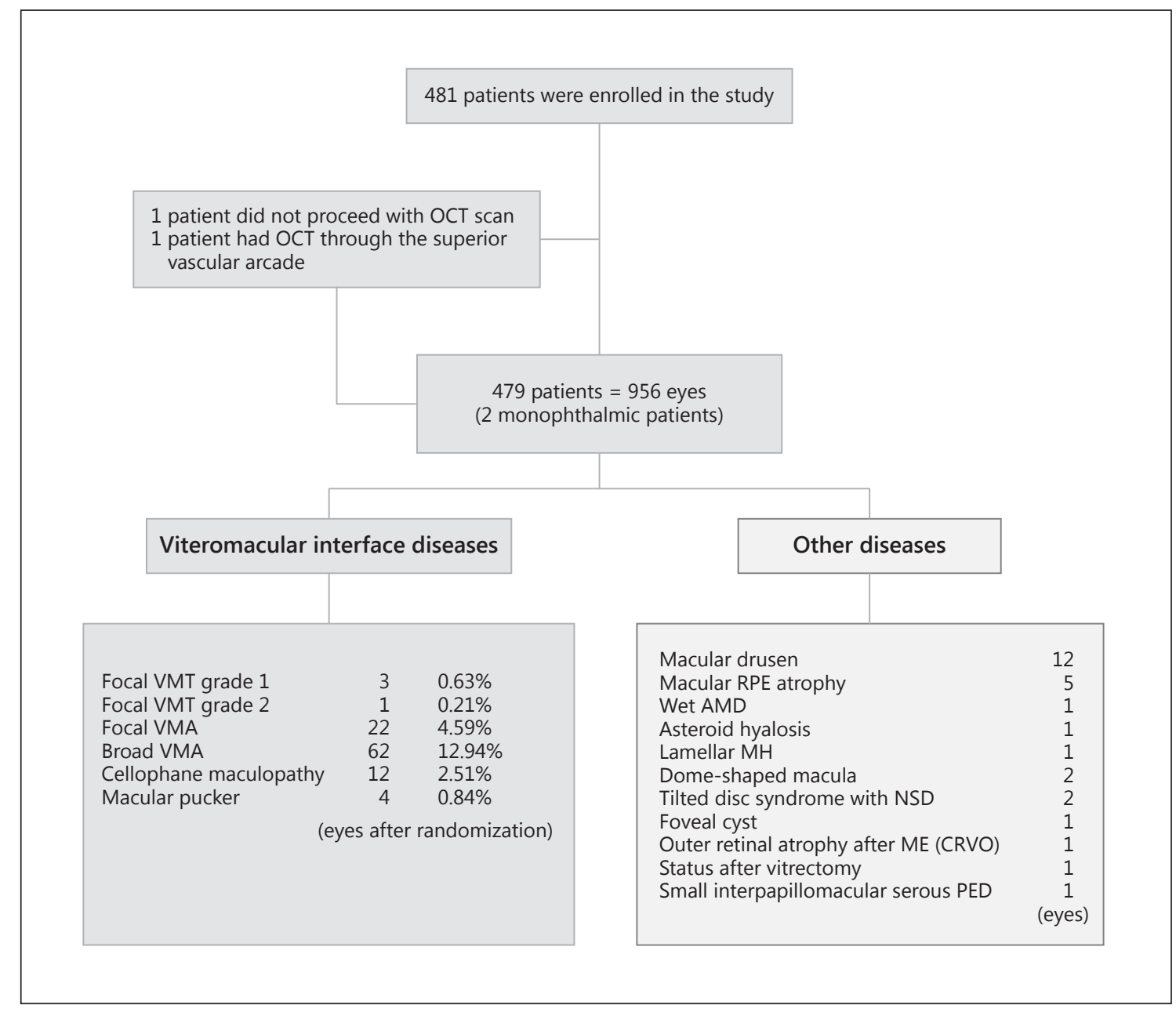

Fig. 1. Diagrama y prevalencia de enfermedades de la IVM en la población total del estudio. RPE = epitelio pigmentario de la retina; $\mathrm{AMD}=$ degeneración macular asociada a la edad; NSD = desprendimiento neurosensorial; $\mathrm{ME}=$ edema macular; $\mathrm{CRVO}=$ oclusión de la vena central de la retina; $\mathrm{PED}=$ desprendimiento del epitelio pigmentario.

$2(0,21 \%), 22$ ojos con AVM focal (4,59\%), 62 ojos con AVM difusa (12,94\%), 12 ojos con maculopatía en celofán $(2,51 \%)$, y 4 ojos con pliegue macular $(0,84 \%)$. La prevalencia estimada en la población belga de TVM focal en grado 1 es del 0,63\% (IC 0,20-1,94), de TVM focal en grado 2 del $0,21 \%$ (IC 0,03-1,48), de AVM focal del 4,59\% (IC 3,02-6,98), de AVM difusa del 12,94\% (IC 10,09-16,60), de maculopatía en celofán del 2,51\% (IC $1,42-4,41$ ), y de pliegue macular del $0,84 \%$ (IC 0,312,22). Del total de 956 ojos, 28 tenían otras anomalías, siendo las más frecuentes las drusas maculares (12 ojos) y la atrofia del epitelio pigmentario de la retina (5 ojos) (fig. 1).
Prevalencia de anomalías en la IVM en la población de estudio de $<50$ años de edad

En la población del estudio con $<50$ años, la prevalencia de AVM focal fue del 0,45\%, de AVM difusa del $7,21 \%$, y del $0 \%$ en los casos de TVM focal de grado 1 , TVM focal en grado 2, maculopatía en celofán y pliegue macular. La prevalencia estimada en la población belga con $<50$ años, fue del 0,45\% para la AVM focal (IC 0,063,20 ), del 7,21\% para la AVM difusa (IC 4,42-11,76) y del $0 \%$ en los casos de TVM focal de grado 1, TVM focal en grado 2 , maculopatía en celofán y pliegue macular. 
Tabla 1. Prevalencia de cambios en la IVM en la población del estudio y prevalencia estimada en la población belga con indicación del IC de Poisson

\begin{tabular}{lccl}
\hline Diagnóstico & Población completa & $<50$ años & $\geq 50$ años \\
\hline TVM focal de grado 1 & $0,63 \%$ (IC 0,2-1,94) & $0 \%$ & $1,17 \%$ (IC 0,38-3,62) \\
TVM focal de grado 2 & $0,21 \%$ (IC 0,03-1,48) & $0 \%$ & $0,39 \%$ (IC 0,05-2,76) \\
AVM focal & $4,59 \%$ (IC 3,02-6,98) & $0,45 \%$ (IC 0,06-3,20) & $8,17 \%$ (IC 5,33-12,53)* \\
AVM difusa & $12,94 \%$ (IC 10,09-16,6) & $7,21 \%$ (IC 4,42-11,76) & $17,9 \%$ (IC 13,41-23,9)* \\
Maculopatía en celofán & $2,51 \%$ (IC 1,42-4,41) & $0 \%$ & $4,67 \%$ (IC 2,65-8,22)* \\
Pliegue macular & $0,84 \%$ (IC 0,31-2,22) & $0 \%$ & $1,56 \%$ (IC 0,58-4,15) \\
\hline
\end{tabular}

* Diferencia estadísticamente significativa entre los grupos de estudio de $<50$ años y $\geq 50$ años.

Prevalencia de anomalías en la IVM en la población de estudio de $\geq 50$ años de edad

En la población del estudio con $\geq 50$ años, la prevalencia de TVM de grado 1 fue del $1,17 \%$, de TVM de grado 2 fue del 0,39\%, de AVM focal fue del 8,17\%, de AVM difusa fue del $17,9 \%$, de maculopatía en celofán fue del $4,67 \%$, y de pliegue macular fue del $1,56 \%$.

En la población belga con $\geq 50$ años de edad, se estima que la prevalencia de TVM de grado 1 es del $1,17 \%$ (IC $0,38-3,62$ ), de TVM de grado 2 del 0,39\% (IC 0,05-2,76), de AVM focal del 8,17\% (IC 5,33-12,53), de AVM difusa del 17.9\% (IC 13,41-23,9), de maculopatía en celofán del $4,67 \%$ (IC 2,65-8,22), y de pliegue macular del 1,56\% (IC $0,58-4,15)$.

Se observó una diferencia estadísticamente significativa en la prevalencia de AVM focal, AVM difusa y maculopatía en celofán entre la cohorte del estudio de $<50$ años y aquellos con $\geq 50$ años. No obstante, en el caso de TVM focal de grado 1 y 2 , el número de sujetos con estas manifestaciones clínicas era demasiado pequeño para detectar una diferencia estadísticamente significativa (tabla 1).

El sexo influyó en la prevalencia de AVM focal: la prevalencia en mujeres $(6,87 \%)$ fue superior que en hombres $(1,84 \% ; \mathrm{p}=0,0085)$.

Se observó que las AVM focal y difusa bilaterales eran más prevalente en visitantes hipermétropes que miopes ( $p=0,0135$ y 0,0317 , respectivamente). Estos datos son congruentes con una mayor prevalencia de DVP incompleta en hipermetropía, tal y como se informó con anterioridad [20].

\section{Discusión}

Los resultados de nuestro estudio son comparables con la prevalencia de TVM previamente publicada en el estudio Beaver Dam Eye Study [21], si los resultados de TVM de grado 1 y 2 se consideran en conjunto para la población con $\geq 50$ años de edad. La prevalencia notificada de la TVM fue del 1,6\%. Este dato se aproxima mucho a los resultados del estudio en la UZ Leuven, que mostró una prevalencia del $1,17 \%$ en el caso de TVM focal de grado 1 y del 0,39\% en el caso de TVM focal de grado 2 . La prevalencia de TVM aumentaba con la edad y era superior en mujeres que en hombres, aunque este dato no resultó estadísticamente significativo [21]. Los resultados de este estudio sobre la prevalencia de TVM no identificaron ninguna diferencia entre mujeres y hombres debido a la limitada cohorte del estudio. El estudio Beijing Eye Study [20] publicó una prevalencia de TVM ligeramente superior, del $2,4 \%$, en una población china de $>50$ años de edad. La prevalencia aumentaba con la edad y era superior en mujeres que en hombres.

A cuatro ojos se les diagnosticó TVM focal de grado 1. En 1 ojo, la agudeza visual estaba limitada debido a un traumatismo craneal; por ello, se eligió un enfoque conservador y el paciente no mostró cambios en el estado de la IVM tras 1 año de seguimiento. En el segundo ojo, se inyectó ocriplasmina 8 meses después sin liberación de la tracción durante el seguimiento a los 6 meses, y la agudeza visual permaneció en 0,9 sin observarse ninguna metamorfopsia significativa (fig. 2a, b). En el tercer ojo, la agudeza visual al inicio fue 1.0 sin metamorfopsia, y se observó una liberación espontánea 2 meses después (fig. 2c, d). El cuarto ojo con TVM focal de grado 1 también mostró una liberación espontánea 6 meses después del estudio.

Ophthalmologica 2017;238(suppl 1):9-15 DOI: $10.1159 / 000477511$ 

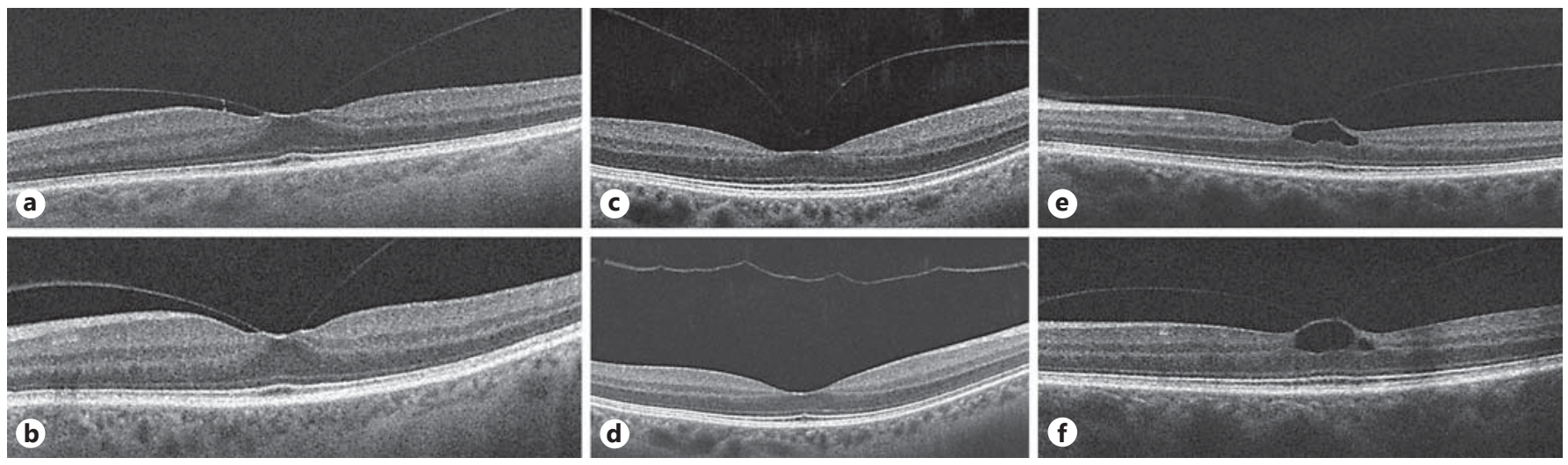

Fig. 2. a, b TVM focal de grado 1 al inicio (a), se inyectó ocriplasmina 8 meses después. En el seguimiento a los 6 meses (b), no hubo ninguna liberación de la tracción. c, d TVM focal de grado 1 al inicio (c); 2 meses después, se pudo observar una liberación espontánea de la tracción (d). e, f TVM focal de grado 2 al inicio (e), no se proporcionó ningún tratamiento. Situación estable en la visita de seguimiento al año siguiente (f).

Un ojo fue diagnosticado con TVM focal de grado 2; la paciente prefirió un enfoque conservador, pues era asintomática, con una agudeza visual de 0,9 . La situación de este caso permaneció estable hasta la visita de seguimiento al año siguiente (fig. 2e, f).

\section{Fortalezas del estudio}

El estudio de la UZ Leuven presenta la prevalencia de todas las subcategorías de AVM y TVM, mientras que en informes anteriores, todas las subcategorías de TVM se consideraron un único grupo.

Este estudio incorporó un sistema de clasificación internacional reconocido (el sistema de clasificación IVTS) y también el uso de un protocolo estándar de OCT (5 exploraciones de alta definición del área foveal) de cada sujeto en la cohorte del estudio.

\section{Limitaciones del estudio}

Aunque el "Day of Science» atrajo a un total de 34.000 visitantes de instituciones y unidades científicas en Flandes y Bruselas, los que visitaron el Departamento de Oftalmología comprendieron un subgrupo del total. El número de participantes en el estudio representa un factor limitador. Puede que muchos de los visitantes hayan presentado un interés personal específico en la oftalmología por su propia experiencia o la de algún familiar.

La etnia de la cohorte del estudio fue predominantemente de raza blanca. Los estudios basados en población de gran tamaño en diferentes grupos étnicos deberían recoger información adicional. Como estudio transversal, no resultó posible describir una relación temporal entre la TVM y el desarrollo de AM de grosor total.

\section{Conclusiones}

En nuestro estudio, se calculó que la prevalencia de cambios vitreomaculares en la población belga de $\geq 50$ años de edad fue del $1,17 \%$ (IC $0,38-3,62$ ) en el caso de TVM focal de grado 1 , del $0,39 \%$ (IC $0,05-2,76$ ) para TVM focal de grado 2, del 8,17\% (IC 5,33-12,53) para AVM focal, y del 17,9\% (IC 13,41-23,9) en el caso de AVM difusa.

El estudio proporcionó datos epidemiológicos sobre alteraciones en la IVM.

Un correcto conocimiento de la epidemiología de alteraciones en la IVM y un diagnóstico temprano permitirán una intervención satisfactoria reduciendo así la incidencia de progresión hacia complicaciones más serias.

\section{Resumen}

Este estudio transversal evaluó la prevalencia de cambios en la interfase vitreoretiniana en el público general. Las anomalías en la interfase vitreoretiniana se diagnosticaron mediante OCT y se les asignaron grados según la clasificación del grupo IVTS. La prevalencia de la TVM fue superior en la cohorte de $\geq 50$ años de edad en comparación con la cohorte de $<50$ años.
Jacob/Stalmans 


\section{Agradecimientos}

A los autores les gustaría agradecer a Zeiss y Thrombogenics por su apoyo logístico en este estudio, así como a todos los 27 empleados y residentes «Day of Science» de 2013.

Erik Spaepen de SBD Analytics, Bekkevoort, Bélgica, llevó a cabo los análisis estadísticos.

\section{Declaración de situación}

El soporte logístico in situ a la hora de realizar este estudio fue proporcionado por Zeiss y Thrombogenics. Los autores tuvieron total libertad editorial para analizar e interpretar sus datos, así como para escribir el artículo. Ninguno de los autores tiene interés comercial. Julie Jacob: gastos de reuniones (Novartis, Bayer, Thea), asesoría (Novartis). Peter Stalmans recibe subvenciones de Thrombogenics NV; honorarios por conferencias de DORC - Dutch Ophthalmic Research Center BV, Ellex y Fluoron, y es asesor de Alcon, Bausch + Lomb, DORC, Haag-Streit y Nano-Retina.

\section{Referencias bibliográficas}

1 Duker JS, Kaiser PK, Binder S, de Smet MD, Gaudric A, Reichel E, et al: The International Vitreomacular Traction Study Group classification of vitreomacular adhesion, traction, and macular hole. Ophthalmology 2013;120: 2611-2619.

2 Gass JD: Stereoscopic Atlas of Macular Diseases: Diagnosis and Treatment, ed 4. St. Louis, Mosby, 1997.

3 Fraser-Bell S, Guzowski M, Rochtchina E, Wang JJ, Michell P: Five-year cumulative incidence and progression of epiretinal membranes. Ophthalmology 2003;110:34-40.

4 Foos RY, Wheeler NC: Vitreoretinal juncture: synchysis senilis and posterior vitreous detachment. Ophthalmology 1982;89:15021512.

5 Sebag J: Age-related differences in the human vitreoretinal interface. Arch Ophthalmol 1991;109:966-971.

6 Johnson MW: Posterior vitreous detachment: evolution and complications of its early stages. Am J Ophthalmol 2010;149:371-382.e1.

7 Krebs I, Brannath W, Glittenberg C, Zeiler F, Sebag J, Binder S: Posterior vitreomacular adhesion: a potential risk factor for exudative age-related macular degeneration? Am J Ophthalmol 2007;144:741-746.

8 Mojana F, Cheng L, Bartsch DU, Silva GA, Kozak I, Nigam N, et al: The role of abnormal vitreomacular adhesion in age-related macular degeneration: spectral optical coherence tomography and surgical results. Am J Ophthalmol 2008;146:218-227.

9 Hikichi T, Fujio N, Akiba J, Azuma Y, Takahashi M, Yoshida A: Association between the short-term natural history of diabetic macular edema and the vitreomacular relationship in type II diabetes mellitus. Ophthalmology 1997;104:473-478

10 John VJ, Flynn HW, Smiddy WE, Carver A, Leonard R, Tabandeh H, Boyer DS: Clinical course of vitreomacular adhesion managed by initial observation. Retina 2014;34:442-446.
11 Hikichi T, Yoshida A, Trempe CL: Course of vitreomacular traction syndrome. Am J Ophthalmol 1995;119:55-61.

12 Charalampidou S, Nolan J, Beatty S: The natural history of tractional cystoid macular edema. Retina 2012;32:2045-2051.

13 Odrobina D, Michalewska Z, Michalewski J, Dziegielewski K, Nawrocki J: Long-term evaluation of vitreomacular traction disorder in spectral-domain optical coherence tomography. Retina 2011;31:324-331.

14 Rahmani B, Tielsch JM, Katz J, Gottsch J, Quigley H, Javitt J, et al: The cause-specific prevalence of visual impairment in an urban population: the Baltimore Eye Survey. Ophthalmology 1996;103:1721-1726.

15 Mitchell P, Smith W, Chey T, Wang JJ, Chang A: Prevalence and associations of epiretinal membranes: the Blue Mountains Eye Study, Australia. Ophthalmology 1997;104:10331040.

16 Wang S, Xu L, Jonas JB: Prevalence of fullthickness macular holes in urban and rural adult Chinese: the Beijing Eye Study. Am J Ophthalmol 2006;141:589-591.

17 Sen P, Bhargava A, Vijaya L, George R: Prevalence of idiopathic macular hole in adult rural and urban south Indian population. Clin Exp Ophthalmol 2008;36:257-260.

18 Klein R, Klein BE, Wang Q, Moss SE: The epidemiology of epiretinal membranes. Trans Am Ophthalmol Soc 1994;92:403-425.

19 Mc Cannel CA, Ensminger JL, Diehl NN, Hodge DN: Population-based incidence of macular holes. Ophthalmology 2009;116: 1366-1369.

20 Shao L, Xu L, You QS, Wang YX, Chen CX, Yang $\mathrm{H}$, et al: Prevalence and associations of incomplete posterior vitreous detachment in adult Chinese: the Beijing Eye Study. PLoS One 2013;8:e58498.
21 Meuer SM, Myers CE, Klein BE, Swift MK, Huang Y, Gangaputra S, et al: The epidemiology of vitreoretinal interface abnormalities as detected by spectral-domain optical coherence tomography. The Beaver Dam Eye Study. Ophthalmology 2015;122:787-795.

22 Stalmans P, Benz MS, Gandorfer A, Kampik A, Girach A, Pakola S, et al: Enzymatic vitreolysis with ocriplasmin for vitreomacular traction and macular holes. N Engl J Med 2012;367:606-615.

23 Jackson TL, Nicod E, Angelis A, Grimaccia F, Prevost AT, Simpson AR, Kanavos P: Pars plana vitrectomy for vitreomacular traction syndrome: a systematic review and metaanalysis of safety and efficacy. Retina 2013; 33:2012-2017.

24 Willekens K, Abegão Pinto L, Vandewalle E, Stalmans I, Stalmans P: Improved efficacy of ocriplasmin for vitreomacular traction release and transient changes in optic disk morphology. Retina 2015;35:1135-1143.

25 Kaiser PK, Kampik A, Kuppermann BD, Girach A, Rizzo S, Sergott RC: Safety profile of ocriplasmin for the pharmacologic treatment of symptomatic vitreomacular adhesion/traction. Retina 2015;35:1111-1127.

26 Nudleman E, Franklin MS, Wolfe JD, Williams GA, Ruby AJ: Resolution of subretinal fluid and outer retinal changes in patients treated with ocriplasmin. Retina 2016;36:738743.

27 Reiss B, Smithen L, Mansour S: Transient vision loss after ocriplasmin injection. Retina 2015;35:1107-1110.

28 Hahn P, Chung MM, Flynn HW, Huang SS, Kim JE, Mahmoud TH, Sadda SS, Dugel PU: Safety profile of ocriplasmin for symptomatic vitreomacular adhesion. Retina 2015;35: 1128-1134

29 World Population Prospects: The 2012 revision. http://esa.un.org/wpp/.
Prevalencia de alteraciones en la interfase vitreoretiniana
Ophthalmologica 2017;238(suppl 1):9-15 DOI: $10.1159 / 000477511$ 